\title{
Data Mining of the Coffee Rust Genome
}

\section{David Botero-Rozo, William Giraldo, Álvaro Gaitán, Marco Cristancho, Diego M. Riaño-Pachón \& Silvia Restrepo.}

\author{
E-Mail: do.botero29@uniandes.edu.co \\ URLs: http://lamfu.uniandes.edu.co/ \\ http://bce.uniandes.edu.co/ \\ http://bioinformatics.cenicafe.org/index.php/wiki
}

\section{WHY DO WE STUDY COFFEE RUST?}

Coffee leaf rust (caused by the fungus Hemileia vastatrix) is the most limiting disease wherever coffee is cultivated. In Colombia, coffee represents $16 \%$ of the country's agricultural GDP and since 2008 high incidence of coffee leaf rust in crops established with susceptible varieties has caused significant reduction in yield. Genome and transcriptome sequencing and bioinformatics analysis tools have been applied for the understanding of this organism and its interaction with the plant and the environmental variations that result in epidemics.

\section{WHAT DID WE GET?}

A total of 73GB of NGS data was generated. An assembly of 396.264 contigs (N50 of 1590 and 841 of mean length) was obtained; this assembly is highly fragmented. Nevertheless, we obtained very large contigs (the largest of $85 \mathrm{~Kb}$ and coverage of $148 \mathrm{x}$ ). After filtering out contigs with putative contaminants with MEGAN (coffee and bacterial sequences that could be into the rust samples), we obtained 31.376 contigs that showed similarities to reported fungal sequences. Forty four putative mitochondrial contigs were identified through blast homologies using Puccinia genome sequences. We also assembled three transcriptomes with a length of 55,791 and 64,752 contigs for non-normalized libraries and 44,297 contigs for a normalized library.



Figure 1. Data cleaning. Here the process of cleaning is illustrated. Top: Every cleaning
step is shown Middle and bottom. lllumina and 454 inputs step is hrown. Middle end bottom: lllumina and 454 inputs and outputs reads given in mil-
lion of reads and fraction of reads with references to the last step. Notet that Illumina reads were not subjected to seq-clean tool (too short for low complexity fitering). The 454 reads were not subjected
complexity masking.


cessful pais.

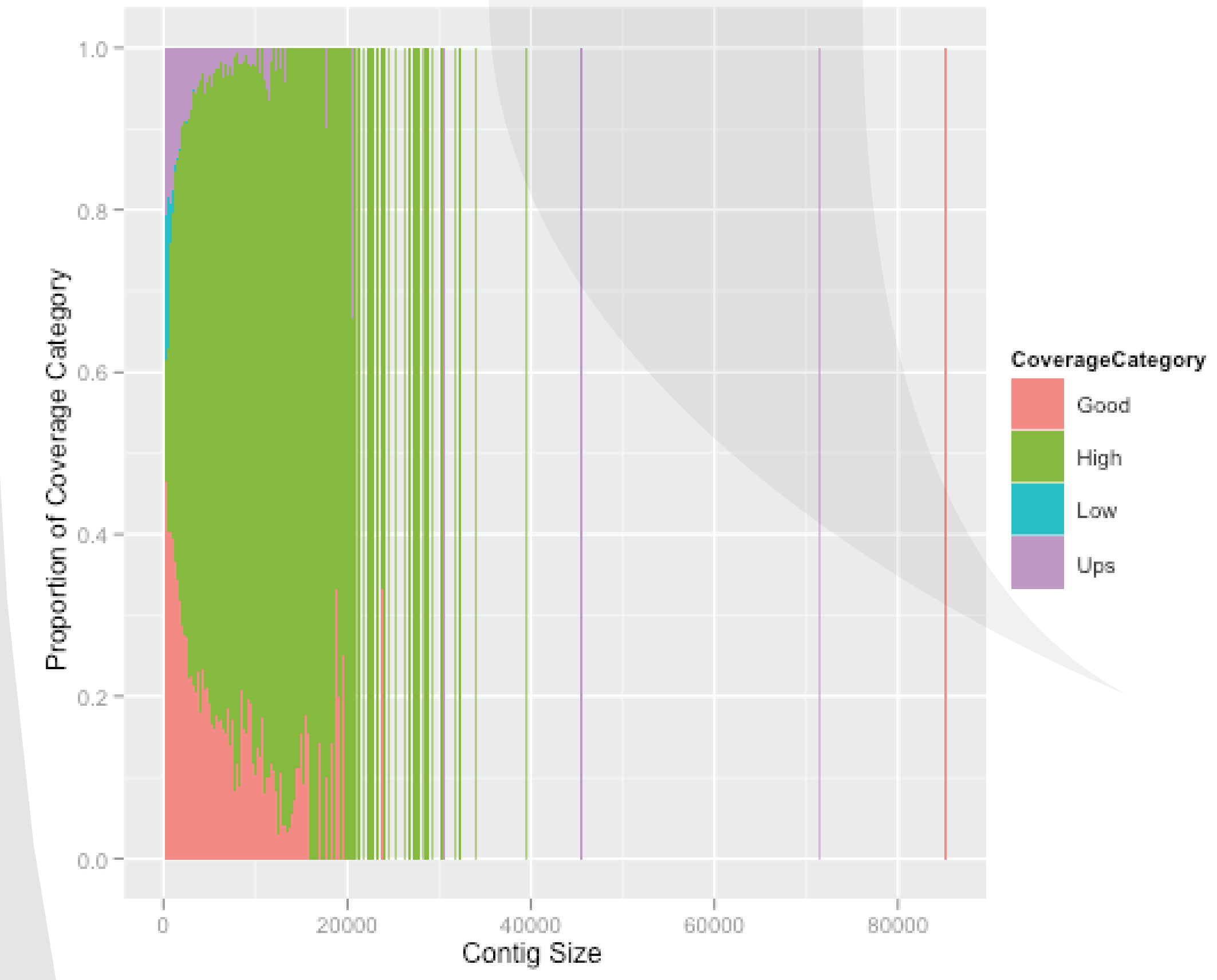

Figure 3. Contigs size fraction of coverage. Results of coverage for the last assem-
bly. Low means coverage smaller than $5 x$, Good is coverage between $5 x$ and $45 x$, High is coverage between $45 x$ and $100 x$ and Ups is coverage higher than $100 x$, these categories are quite arbitrary. The bins for the contig size are $150 \mathrm{bp}$ wide. The averag
coverage of a considerable fraction of the contigs of this assembly is acceptable (those with High and Good values in the Figure.

\section{WHAT DID WE DO?}

We sampled isolates from field crops and collected eight different races of $H$. vastatrix. One isolate per race was sequenced by Illumina and 454 technologies. The data was subjected to pre-quality control with FastQC and was cleaned. After testing several assemblers with different combinations of data, we decided to do a hybrid assembly using the CLC assembler. The assembly was analyzed with MEGAN to evaluate the level of contamination and do a first approximation to the biological communities associated to $H$. vastatrix on the coffee leaf. BLAST was used to search the $H$. vastatrix mitochondria, comparing our contigs against the Puccinia mitochondrial genome (the closest sequenced organism to $H$. vastatrix). The mitochondrial contigs identified were annotated with MAKER. Finally, using Trinity we assembled three transcriptomes from different races of $H$. vastatrix.

This is the first approach to study the genome of the causal agent of the coffee rust, $H$. vastatrix. Further data mining will allow the identification of virulence and aggressiveness factors, important in the characterization of new races of the pathogen and the detection of isolates that might infect resistant coffee varieties. 